\title{
América Latina: Militares y Política después del fin de la Guerra Fría ${ }^{1}$
}

\author{
Alberto Sepúlveda Almarza²
}

\begin{abstract}
Resumen
La monografía aborda los cambios de América Latina después del fin de la Guerra Fría. Se describe el cuadro existente en el pasado y se señalan algunas iniciativas y tendencias del presente, como la democratización y las labores de cooperación entre fuerzas armadas. Se analizan los casos de presencia de la política militar en Perú con Fujimori, Venezuela con Hugo Chávez, Bolivia con Evo Morales y la transición iniciada en Cuba por Raúl Castro.
\end{abstract}

Palabras clave: Política militar, fuerzas armadas, democratización.

\begin{abstract}
This monograph tackles changes in Latin America after the end of the Cold War. It describes the past panorama and points out some present initiatives and trends such as democratization and cooperative actions among the armed forces. The text analyses cases of militarized politics in Peru with Fujimori, in Venezuela with Hugo Chávez, in Bolivia with Evo Morales and the Cuban transition started by Raúl Castro.
\end{abstract}

Key words: militarized politics; armed forces; democratization

\begin{abstract}
Resumem
A monografia aborda as mudanças de America Latina depois do fim da Guerra Fria. Descreve-se o quadro existente no passado e se sinalam algumas iniciativas e tendências do presente, como a democratização e as labores de cooperação entre forças armadas. Analisam-se os casos de presença da política militar em Peru com Fujimori, Venezuela com Hugo Chávez, Bolivia com Evo Morales e a transição iniciada em Cuba por Raúl Castro.
\end{abstract}

Palavras chave: Política militar, forcas armadas, democratização.

\footnotetext{
Artículo recibido el 5 de septiembre de 2013 y aceptado el 30 de septiembre de 2013.

2 Instituto de Relaciones Internacionales de la Universidad de Viña del Mar (Chile).

E-mail: alberto.sepulveda.almarza@gmail.com
} 
En los años de la Guerra Fría el panorama planetario estaba caracterizado por el Sistema Bipolar que consistía básicamente en un modelo sustentado en la hegemonía política y militar de Estados Unidos y la Unión Soviética. Por la imposibilidad de definir sus diferencias por medio de la guerra, gracias al empate nuclear, los contendientes lucharon, en gran medida, usando métodos indirectos y con gran influencia de los servicios de inteligencia. Es así que surgieron guerras no declaradas, calificadas como "conflictos de baja intensidad" en las cuales un ejército de una superpotencia o de un aliado combatía contra otro "irregular" abastecido y entrenado por la otra. Este tipo de encuentros se dio en Vietnam, Afganistán, Angola y Centroamérica para citar algunos casos $^{3}$.

Los bandos armados irregulares se llamaban "Ejércitos de Liberación Nacional" si eran organizados por Moscú y "Luchadores de la Libertad" si lo hacía Washington.

\section{El panorama latinoamericano}

Estados Unidos estableció, con posterioridad a la II Guerra Mundial, un ordenamiento jurídico para consolidar la alianza o dependencia de los países latinoamericanos. En el marco político surgió la Organización de Estados Americanos (OEA) y en el militar el Tratado Interamericano de Asistencia Recíproca (TIAR) y la Junta Interamericana de Defensa. Además existían Tratados de Asistencia Mutua, que eran acuerdos bilaterales de alianza militar entre EE.UU y un país latinoamericano. Todos los documentos señalaban la colaboración frente a una "agresión extra-regional", que tenía su origen en el caso de Alemania, durante la I y II Guerra Mundial, y en el período de la Guerra Fría, en la Unión Soviética.

El fundamento ideológico de la alianza era el modelo liberal, es decir, democracia y economía de mercado, que contrastaba con el totalitarismo político y la economía centralmente planificada de la URSS y sus satélites.

Sin embargo, no hubo mucho apego a los aspectos democráticos, por parte de Washington, y entre 1948 y 1982 promovió golpes de Estado en varios países de la Región. A contar de 1982 se va a abandonar la alternativa dictatorial debido a dos situaciones. La primera fue la Guerra de las Malvinas, en la cual una dictadura pro EE.UU., la argentina, desató una aventura militar contra Gran Bretaña, el aliado por excelencia de la Casa Blanca, para apoderarse de las Islas Malvinas, una estratégica zona, desde la cual se controla el tráfico marítimo y aéreo entre América del Sur, la Antártida

3 Sobre la Guerra de Vietnam ver Kissinger (1979: 893-1010) y Nixon (1990: 358-367). 
y África. Obviamente que el Pentágono no iba a permitir la humillación de Gran Bretaña por los políticamente inestables argentinos y mucho menos aceptar el cambio de posesión de las estratégicas Islas Malvinas (Haig, 1984: 298-345; West, 1997).

El segundo factor se dio con las guerras centroamericanas entre 1979 y los años 1980. Allí se enfrentaron ejércitos e irregulares financiados por Washington y Moscú (Haig, 1984: 130-158), en este último caso vía La Habana. Los elementos doctrinarios que servían de fundamento propagandístico al conflicto eran la democracia por parte de los que se encontraban a favor de Estados Unidos y la Revolución Antiimperialista y Popular, por parte de los simpatizantes cubanos.

En estas condiciones, a contar de 1982, Washington dejó de apoyar a los gobiernos militares y, por el contrario, inició una presión en torno a la democratización y la entrega del poder a los civiles. Esta línea subsiste hasta nuestros días.

\section{Estados Unidos y los militares latinoamericanos}

En el siglo XIX el proceso de profesionalización de los ejércitos de América del Sur se dio por medio de misiones militares de Francia y Alemania principalmente, aunque también hubo colaboración menor de Italia. En el caso de Centroamérica y el Caribe la labor de formación estuvo a cargo de EE.UU.

La desaparición de los países europeos, como grandes potencias, después de la Segunda Guerra Mundial, implicó que EE.UU quedó como el actor dominante en todo el continente. Como consecuencia los sistemas de entrenamiento, las doctrinas bélicas y el armamento provenían de Estados Unidos. Sólo Argentina en los primeros gobiernos de Juan Domingo Perón (1946-1954) resistió ésta tendencia, pero con posterioridad al derrocamiento del líder justicialista se sumó a la subordinación al Pentágono (Rapoport, 2005: 309-415).

La situación comienza a cambiar con la Revolución Cubana que llevó, en la década de 1960, a un intenso proceso de armamento y a la creación de unas Fuerzas Armadas modernas y profesionales bajo la égida de Moscú. Incluso unidades del Ejército, Marina y Fuerza Aérea de la URSS se instalaron en Cuba. Este proceso se incrementa después del fracaso de la invasión de Bahía Cochinos en 1961, operación propiciada por la CIA para derrocar a Fidel Castro (Wiener, 2008: 175-217).

El poderío de las fuerzas armadas de Cuba llega a tal nivel que, incluso, fueron utilizadas por Moscú en una serie de guerras africanas (Benemelies, 1988) en la década de 1970. Los cubanos, además, entrenaron a grupos armados irregulares que combatieron en Nicaragua, logrando el triunfo, 
en El Salvador contra ejércitos profesionales y en Chile contra el gobierno militar de Pinochet.

En 1968 toman el poder los militares en Perú y ante la negación de armamentos de EE.UU desatan un importante programa de modernización con material soviético, especialmente en blindados y aviación. Para enseñar el uso de los sofisticados armamentos llegaron instructores soviéticos y cubanos.

A contar de la década de 1970, EE.UU. se centró en las guerras de Vietnam y países vecinos y disminuyó considerablemente la colaboración militar y el abastecimiento de armas a América Latina. Como consecuencia el vacío fue llenado en América del Sur por Francia, Alemania, Italia, Gran Bretaña, España e Israel. Y surgió, asimismo, una importante industria militar especialmente en Argentina, Brasil, Chile y, en menor grado, en Perú.

\section{Dictaduras y Derechos Humanos}

Estados Unidos, como todos los países del mundo, es una constelación de distintas ideologías y proyectos estratégicos. Una visión bastante simplista en algunos medios latinoamericanos tiende a suponer que en EE.UU existe una sola postura política, basada en la dominación sobre los más débiles, usando métodos despiadados.

EE.UU. mantiene desde hace décadas diversos enfoques en su política exterior que van desde al aislacionismo hasta la intervención incluso sustentada en fundamentos casi religiosos.

Hay dos visiones dominantes en lo referente a su actuación en los países desarrollados. La primera plantea que EE.UU debe ser modelo por sus valores liberales que incluye a la democracia y a los Derechos Humanos. Ambas ideas que se encuentran desde la Independencia de Gran Bretaña, en el siglo XVIII, están estampadas en la Constitución y son la base del sistema político.

Hoy se llama "poder blando" al valor que se atribuye a EE.UU como centro de influencia mundial por el atractivo ideológico de sus instituciones democráticas y su cultura de masas, expresada en el cine, en la literatura y su música ${ }^{4}$.

Hay otra visión para lidiar con los movimientos nacionalistas, revolucionarios y "fundamentalistas" opuestos a la hegemonía de EE. UU y su cultura.

Estas tesis surgieron entre intelectuales en temas de seguridad franceses. Después de la derrota en Indochina, en la década de 1950, se planteó que ella

4 Joseph Nye desarrolló el concepto del "poder blando" en su libro The paradox of American power: why the world only superpower can't go it alone (2002). 
se debía a que los comunistas desarrollaron un tipo de guerra revolucionaria, basada en conceptos propios de la guerrilla y con énfasis en el apoyo civil, la motivación de los combatientes y en una organización clandestina. Para destruir la maquinaria política y militar del adversario había que quebrarlo psicológicamente no sólo para que dejaran de luchar sino, incluso, para que delataran a sus camaradas (Robin, 2005).

Ello llevó a una nueva concepción intelectual de la lucha armada que abandona las reglas de la Convención de Ginebra y que preconiza la tortura y la violencia a los civiles, incluso a los no combatientes, ya que no se sabe quién es espía del enemigo, miembro del ejército irregular, cuadro político o simplemente un civil al margen del conflicto ${ }^{5}$.

Las tesis de Indochina se aplicaron en los años 1960 en la rebelión de Argelia y se practicó la tortura y las desapariciones en forma usual. Posteriormente las tesis francesas se exportaron a EE.UU donde fueron adoptadas por el Pentágono, la CIA y demás Servicios de Inteligencia.

Estas ideas fueron traídas a América Latina tanto por asesores estadounidenses como franceses y fueron usadas por las dictaduras militares y sus Servicios de Inteligencia, como fueron denominadas organizaciones, como la chilena de la primera parte del gobierno de Pinochet. Fue el caso de la Dirección de Inteligencia Nacional (DINA)

En estas condiciones llegaron a la Región dos visiones ideológicas antagónicas y ambas propiciadas por centros de poder de Estados Unidos. Una enfatizaba el uso de tortura, incluso, psicológica, para destrozar la capacidad de los movimientos revolucionarios, comunistas, castristas o simplemente aliados de la otra superpotencia.

La segunda visión enfatizaba los valores morales, civilizados y moderados de EE.UU. De ahí la importancia del respeto a los Derechos Humanos. Cabe recordar que la Declaración Universal de los Derechos Humanos, que forma parte del Sistema de Naciones Unidas, fue propiciada por EE.UU. a instancias de Eleanor Roosevelt.

Washington ha promovido los Derechos Humanos desde el Congreso, por ejemplo, la Enmienda Kennedy, del senador Ted Kennedy que prohibió el abastecimiento de armas al Gobierno de Pinochet, o por la Casa Blanca como ocurriera durante el gobierno Carter. El tema de los Derechos Humanos se incluyó en las negociaciones con la URSS (Judt, 2006).

5 Un buen análisis sobre las tácticas terroristas lo realiza Villamizar (2007).

6 Kornbloch (2004: 113-137). 
Es así que simultáneamente EE.UU usó dos planteamientos doctrinarios y estratégicos radicalmente distintos en sus relaciones con los gobiernos de América Latina, especialmente los de origen castrense.

A contar de 1982 se ha enfatizado la postura liberal y hoy se dictan cursos de Derechos Humanos en los institutos militares de América Latina y la política oficial de Washington es de rechazo a los golpes de Estado en la región.

Hay que recalcar que durante la Guerra Fría hubo fuertes enfrentamientos entre el Congreso de EE.UU. y la Casa Blanca en lo relativo a los métodos y a la magnitud de las operaciones de la CIA. Y ello llevó a la "Operación IránContras" en la cual el Pentágono y la CIA cooperaron con los gobiernos de Irán, Israel y Arabia Saudita, todos enemigos presuntamente irreconciliables, para abastecer de armas a Irán y a los "contras", las organizaciones irregulares que combatían al gobierno procastrista de Nicaragua. En Honduras una misión militar argentina entrenaba en métodos de tortura a ejércitos y grupos irregulares centroamericanos (Weiner, 2008: 421-429).

EE.UU. en la actualidad tiene otra vez, una dualidad de enfoques frente a la tortura y los Derechos Humanos.

En América Latina se preconiza la democracia y el respeto a los Derechos Humanos. Pero en Medio Oriente se aplica la Doctrina Francesa y en Guantánamo los presos políticos no tienen mayores garantías. Y por otra parte la CIA públicamente preconiza la utilidad de las "torturas psicológicas".

Obviamente el debate entre ambas posturas sigue en EE.UU. Sin embargo, con posterioridad a los atentados a las Torres de Manhattan en el 2001, un importante sector de la opinión pública de EE.UU piensa que a los terroristas no se les pueden aplicar las garantías de un Estado de Derecho.

\section{Los militares latinoamericanos durante la Guerra Fría}

En esos años el papel principal de las Fuerzas Armadas estuvo centrado en las siguientes ideas:

- La defensa de las fronteras

Esta concepción venía del siglo XIX y fue consecuencia de la Independencia de las colonias de España y Portugal. La falta de una claridad en lo

7 Un buen análisis sobre la política exterior de Estados Unidos después del 11 de septiembre de 2001 lo realiza Zeraoui (2008). Ver diario El Mercurio, Santiago de Chile, 19 de julio de 2009, p. A-8, "Los problemas de Inteligencia que obligaron al Presidente Obama a mirar atrás" y diario La Tercera, Santiago de Chile, 14 de julio de 2009, p. 10: "Bush dio luz verde a CIA para asesinar a líderes de Al Qaeda". Ver también Souaidia (2002). 
que respecta a los límites coloniales llevó a múltiples disputas entre Estados vecinos y algunas se solucionaron por la guerra.

Las fuerzas armadas latinoamericanas tenían su razón de ser en la capacidad de enfrentar con eficacia al poder militar de un país vecino.

Durante la Guerra Fría, EE.UU. consolidó la paz en el continente, garantizó el respeto al statu quo fronterizo, no sólo en América sino también en Europa, para impedir un conflicto con la URSS. Sólo en 1971 se desató un breve choque armado entre Ecuador y Perú en la Cordillera del Cóndor.

La situación más tensa la vivió Chile, que en 1975 fue amenazado por Perú, armado por los soviéticos, y en 1978 por Argentina. La presión de Washington fue determinante para disuadir a los militares peruanos y argentinos ${ }^{8}$.

En el primer caso el belicoso General Juan Velasco Alvarado fue depuesto por el moderado General Francisco Morales Bermúdez; en el segundo se llegó a una solución mediante la intervención del Papa Juan Pablo II. Cabe recordar que existió una estrecha alianza desde 1947 entre el Gobierno de EE.UU. y el Vaticano, ambos fervientes anti-comunistas.

En 1982 los militares argentinos, desoyendo los consejos del Secretario de Estado de EE.UU. Alexander Haig, se negaron a poner fin a su invasión a las Islas Malvinas. En la consecuente guerra la Casa Blanca dio un fuerte apoyo a Gran Bretaña (Haig, 1984: 135-150).

- Las fuerzas armadas constituyen la reserva frente a procesos políticos que puedan dar origen a situaciones revolucionarias o favorables a los comunistas.

Desde los años 1920 los estamentos castrenses latinoamericanos fueron fuertemente anti-comunistas tanto por intentos de revueltas internas amparados bajo los conceptos marxistas-leninistas o por la prédica estadounidense llevada a cabo en centros académicos, instituciones especializadas como la Escuela de las Américas (Gill, 2005) o por un amplio número de libros o revistas como "Military Review". No hay que olvidar que el Pentágono otorgaba armas y entrenamiento a los latinoamericanos y que la CIA tuvo un papel muy importante en la generación y desarrollo de los Servicios de Inteligencia de la Región (Dinger, 2004).

8 Según el Asesor de Prensa del Presidente Juan Velasco Alvarado la orden de invadir la ciudad de Arica, Chile, por tropas peruanas estuvo fijada para el día 06 de agosto de 1975. El 29 de agosto el Gral. Francisco Morales Bermúdez se sublevó en Tacna y depuso al Gral. Veloso Alvarado. Ver Diario La República, Lima, 27 de septiembre de 1995, "Perú y Chile al borde de la guerra: 06 de agosto de 1995". 
Es así que la Doctrina de Seguridad Nacional, basada en las tesis de EE.UU., fue el fundamento ideológico de varios gobiernos militares ${ }^{9}$.

Ello no implica desconocer que hubo desavenencias entre Washington y varios regímenes castrenses. El más importante fue con Juan Velasco Alvarado en Perú, René Barrientos en Bolivia, con los gobiernos del "Milagro económico" de Brasil que aspiraban a convertir a su país en una potencia mundial y, por ende, competitiva con EE.UU. En Argentina y Brasil los militares desarrollaron programas que llevaban al armamento nuclear, en los años 1960 y 1970, en franco desafío a Washington.

- Las Fuerzas Armadas están más capacitadas que los políticos civiles para modernizar y desarrollar a los países latinoamericanos.

Esta concepción que viene de la lucha entre caudillos militares y oligarquías civiles, desde el siglo XIX se repite de tiempo en tiempo en la Región.

En el fondo ha sido el fundamento de todas las dictaduras militares en el continente. Estas ideas han sido recogidas por muchos movimientos revolucionarios que preconizan la lucha armada y que cuando conquistan el poder se apoyan en los nuevos ejércitos regulares y sus líderes siempre visten uniforme militar. Es el caso, por ejemplo, de castristas y sandinistas.

En nuestros días el régimen instalado por Hugo Chávez en Venezuela tiene estos fundamentos: el Ejército es más honesto, capaz y compenetrado con el pueblo que las oligarquías políticas civiles (Garay, 2009).

\section{Post Guerra Fría (1989- )}

En el mundo postguerra fría se han producido las siguientes situaciones:

Revisión de la participación política de los militares

Tal como se señaló, a contar de los años 1982 Estados Unidos promovió la democratización de los gobiernos de la Región y obviamente el fin de las dictaduras, civiles o castrenses.

Se han producido, sin embargo, nuevos cuadros de actividad militar. Los principales son los siguientes casos:

- Perú

En la década de 1990 arreciaba la guerra revolucionaria promovida por el movimiento guerrillero "Sendero luminoso". En esas condiciones el Presidente

9 Una visión moderna de la Doctrina de Seguridad Nacional se encuentra en Needler (2009). 
Alberto Fujimori dio un golpe de Estado apoyado por las fuerzas armadas. Después de intensas negociaciones con la Organización de Estados Americanos (OEA) se volvió a la democracia pero con profundos cambios legales que profundizaron el poder de la Presidencia.

Fujimori gobernó respaldado principalmente por el Ejército, derrotó a Sendero Luminoso (Jara, 2003), logró acuerdos en las fronteras con Chile y Ecuador y estableció un modelo económico sustentado en la globalización que permitió altas tasas de crecimiento del PIB.

Terminados estos procesos tuvo que renunciar por grandes protestas populares que lo acusaban de corrupción, de dictador y de violador de los Derechos Humanos.

- Venezuela

A fines de los años 1990 triunfó en las elecciones presidenciales el coronel (R) Hugo Chávez, quien en el pasado había promovido un golpe de Estado contra el presidente Carlos Andrés Pérez acusándolo de corrupción e incompetencia. El golpe fracasó pero lanzó a la fama al coronel.

Chávez ha reformado la Constitución estableciendo la "República Bolivariana de Venezuela" y ha asumido la categoría de centro dominante del sistema político de su país.

Su principal apoyo organizado son las fuerzas armadas, en especial, el Ejército (Ramos Álvarez, 2009).

Sus años de gobierno se han visto favorecidos por altos precios de petróleo lo que ha permitido a Chávez contar con enormes recursos para gastar en los sectores más modestos e intervenir en otros países apoyando programas sociales y educacionales y financiando candidaturas presidenciales.

Chávez ha establecido alianzas con Cuba, Argentina, Bolivia, Nicaragua y Ecuador, otorgando ayuda financiera o recursos petroleros, servicios médicos o armamento militar a los gobiernos de esos países.

El estilo de Chávez es de confrontación verbal con Estados Unidos y contra el capitalismo, promoviendo la revolución socialista. Sin embargo los cambios en el sistema económico han sido cosméticos y se mantiene el apoyo al empresariado local. Hay, eso sí, confrontaciones con el capital extranjero y se sigue vendiendo petróleo a EE.UU.

Se ha mencionado que el Ejército juega un papel moderador y que presionó para que Chávez aceptara los resultados adversos de un plebiscito que permitía su reelección indefinida y asimismo el retiro de algunas propuestas impopulares como la Ley de Inteligencia. 


\section{- Cuba}

Fidel Castro va desapareciendo de la escena y su hermano Raúl ha asumido la Presidencia.

El castrismo se apoyó en la burocracia del Partido Comunista y en las Fuerzas Armadas. Raúl Castro, comandante en jefe del Ejército, tiene a su cargo el delicado proceso de transición política y para ello es fundamental el apoyo del Ejército que, obviamente, está jugando un papel político relevante.

- Bolivia

Evo Morales, el actual presidente, está tratando de impulsar un sistema político en el cual los indios aymaras y, en menor medida, los quechuas, asuman un papel dominante.

Morales está enfrentando un cuadro muy complejo en el cual existe una fuerte oposición de las clases altas y medias del Altiplano, de las ricas regiones de la "Medialuna", Pando, Santa Cruz, Beni y Tarija que rechazan la hegemonía aymara y del Altiplano.

El conflicto se ha radicado en el proyecto de Constitución patrocinado por el Gobierno. Morales ha tenido que respaldarse en el Ejército, última estructura que garantiza la unidad del país.

Es sintomático que para el cargo de Cónsul General en Chile, se designara en 2008 al anterior comandante en jefe del Ejército. El consulado, que hace las veces de Embajada ante la ausencia de relaciones diplomáticas entre La Paz y Santiago, es un cargo de especial relevancia para las negociaciones marítimas entre Chile y Bolivia.

\section{La Diplomacia Militar}

Este término fue acuñado por el Almirante Massera de Argentina, en los años 1970 para justificar el control de los Ministerios de Relaciones Exteriores por parte de los militares. Fenómeno que se dio en Argentina, Chile y Perú: según Massera el lenguaje directo y las negociaciones francas entre los militares eran más eficientes que el rebuscado lenguaje y el estilo dilatorio de los diplomáticos de carrera. El modelo se hundió con la Guerra de Malvinas (Costa Méndez, 1993).

En nuestros días estamos observando un intenso acercamiento entre las fuerzas armadas latinoamericanas, incluidas las mexicanas. Es frecuente que en las Academias Militares participen alumnos y profesores de los diversos estamentos castrenses de la Región, que en los viajes de los buques escuela participen cadetes navales de varias nacionalidades. El intercambio mayor se está dando entre Argentina, Bolivia, Chile y Perú. 
- El fin del control de las superpotencias en la Región.

El término de la Guerra Fría llevó a la desmembración de la Unión Soviética (URSS) y con ello el término de la posibilidad de un enfrentamiento armado entre las fuerzas armadas de Estados Unidos y la URSS.

En la actualidad no se avizora un peligro de guerra entre las grandes potencias y lo que hay es un combate contra el terrorismo o guerras irregulares.

El fin de la URSS llevó a la imposibilidad de que Cuba siguiera promoviendo guerrillas en América Latina y Fidel Castro, desde los años 1990, fue desmantelando los grupos insurgentes y buscó el restablecimiento de relaciones diplomáticas con los gobiernos de la Región.

Estados Unidos, por su parte, está comprometido en el Medio Oriente y sus ojos se fijan en países como Afganistán, Irak o Irán, no en América Latina.

En este panorama surge el fenómeno novedoso que la Región se encuentra libre de hegemonías en los sectores castrenses de EE.UU. y que Cuba no depende de la URSS.

Esta situación coloca en entredicho los vínculos especiales con Estados Unidos como el TIAR y la Junta Interamericana de Defensa. México a pesar de que tiene un Tratado de Libre Comercio con EE.UU. y que su comercio exterior es de un $80 \%$ con sus vecinos del norte, se ha retirado de la Junta Interamericana de Defensa.

- Nuevas iniciativas.

En la actualidad las fuerzas armadas latinoamericanas gozan en general, de alto prestigio y popularidad en las poblaciones de la Región, en momentos, en que la delincuencia aumenta (Zaverucha, 2009).

Como consecuencia existe una presión para que los sectores castrenses apoyen a las policías en la lucha contra la criminalidad. Esta situación se ha dado en Brasil donde el Ejército ha ocupado, en más de una oportunidad, las "favelas" más peligrosas y controladas por narcotraficantes. En México la lucha contra los "carteles de la droga" está siendo dirigida por el Ejército.

En América del Sur se han venido tomando medidas, desde los años 1980, para establecer un sistema de colaboración entre las fuerzas armadas. Esto se inició con el Mercado Común del Sur (MERCOSUR) que estableció un sistema de cooperación.

En los últimos tiempos se aprobó la creación de la Unión de Naciones de América del Sur (UNASUR) y existe en estudio una propuesta de Brasil para establecer una especie de Comunidad de la Defensa que coordine las labores en especial en las selvas del interior, el conjunto Orinoco, Amazonas, Paraná. 
Esta región puede ser usada por narcotraficantes, guerrilleros, organizaciones criminales y, además, existe el temor de Brasil a iniciativas de ONG de EE.UU. que en más de una ocasión han propiciado la "internacionalización de la Amazonia".

La propuesta del gobierno de Bush de activar una IV flota para operar en América Latina aumentó los temores de Brasil.

Varios países sudamericanos han enviado a contingentes de sus fuerzas armadas a lugares conflictivos ya sea para mantener el orden o imponer la paz, bajo el alero de las Naciones Unidas. Argentina, Brasil, Chile, Perú y Uruguay han destacado en estas tareas.

Existe incluso una brigada, la "Cruz del Sur", con tropas y mando binacional entre Argentina y Chile y es posible que en el futuro se negocie algo parecido con Perú y Brasil.

La intervención más destacada es en Haití, bajo el mando de un general brasileño y tropa de varios países sudamericanos y de continentes de otras regiones del mundo (Bigatao, 2009).

\section{Conclusiones}

- El término de la Guerra Fría ha llevado a cambios de consideración en América Latina entre los cuales podemos señalar:

- El establecimiento de un consenso en materia económica (economía de mercado o de libre empresa) y política (democracia).

- Reducción de los conflictos fronterizos y uso de la diplomacia, no de la amenaza de guerra, para negociar los desacuerdos.

- Término de la presencia de la URSS y declinación de la presencia de EE.UU. Este cuadro lleva a una reducción del papel tradicional de influencia política de los militares e incluso a rebajas en los gastos de defensa (Deare, 2009).

- Estamos viviendo un proceso de ajuste de las sociedades latinoamericanas ante las nuevas realidades y ello ha llevado a una creciente colaboración entre los estamentos militares de la Región (Bartolomé, 2009).

- En aquellos países donde existe un mayor grado de incertidumbre o conflicto debido a las propuestas de reformas intensas los militares ocupan un creciente espacio político. Es el caso de Bolivia y Venezuela en la actualidad, el Perú de Fujimori en el pasado y en la Cuba de la transición política de nuestros días (Alda Medias, 2009). 
- Uno de los instrumentos de inserción internacional de las Fuerzas Armadas es su participación en las Misiones de Paz, bajo el alero de las Naciones Unidas (Bartolomé, 2006).

- Se ha llegado a la creación de un Consejo de Defensa de América del Sur, promovido por Brasil e influido por la experiencia de la Unión Europea.

\section{Referencias bibliográficas}

ALDA MEDIAS, S. (2009). National development: the main contribution of armed forces in the new left-wing governments. In: INTERNATIONAL POLITICS SCIENCE ASSOCIATION, RESEARCH COMMITTEE. Armed Forces and Society: new challenges and environments. Santiago de Chile: Ed. Centro de Estudios e Investigaciones Militares (CESIM), pp. 103-110.

BARTOLOMÉ, M.C. (2006). La seguridad internacional post 11/9: contenidos, debates y tendencias. Buenos Aires: IPN Editores, Instituto de Publicaciones Navales, pp. 271-291.

BARTOLOMÉ, M.C. (2009). Singularities of the South American armed forces explanations ands repercussions. In: INTERNATIONAL POLITICS SCIENCE ASSOCIATION, RESEARCH COMMITTEE. Armed Forces and Society: new challenges and environments. Santiago de Chile: Ed. Centro de Estudios e Investigaciones Militares (CESIM), pp. 228-231.

BENEMELIES, J. F. (1988). Castro, subversión y terrorismo en África. Madrid: Ed. San Martín, pp. 241-431.

BIGATAO, J. DE P. (2009). Brazil and the United Nations peacekeeping operations: the case of Haiti. In: INTERNATIONAL POLITICS SCIENCE ASSOCIATION, RESEARCH COMMITTEE. Armed Forces and Society: new challenges and environments. Santiago de Chile: Ed. Centro de Estudios e Investigaciones Militares (CESIM), pp. 475-485.

COSTA MÉNDEZ, N. (1993). Malvinas: esta es la historia. Buenos Aires: Ed. Sudamericana, pp. 157-240.

DEARE, C. A. (2009). Improving American Defense Policy Toward Latin American. In: INTERNATIONAL POLITICS SCIENCE ASSOCIATION, RESEARCH COMMITTEE. Armed Forces and Society: new challenges and environments. Santiago de Chile: Ed. Centro de Estudios e Investigaciones Militares (CESIM), pp. 159-167.

DINGER, J. (2004). Operación Cóndor: una década de terrorismo internacional en el Cono Sur. Santiago de Chile: Ed. B.S.A., pp. 103-122.

GARAY VERA, C. (2009). The ideological environment of the bolivarian defense. In: INTERNATIONAL POLITICS SCIENCE ASSOCIATION, RESEARCH COMMITTEE. 
Armed Forces and Society: new challenges and environments. Santiago de Chile: Ed. Centro de Estudios e Investigaciones Militares (CESIM), pp. 407-420.

GILL, L. (2005). Escuela de las Américas: entrenamiento militar, violencia política e impunidad en las Américas. Santiago de Chile: Ed. LOM, 2005, pp. 125-197.

GUNDER FRANK, A. (1978). Crítica y anticrítica: ensayo sobre la dependencia y el reformismo. Madrid: Ed. Zero, pp. 22-37.

HAIG, A. (1984). Memorias. Buenos Aires: Ed. Atlántida, pp. 298-345.

JARA, U. (2003). Ojo por ojo: la verdadera historia del Grupo Colina. Lima: Grupo Editorial Norma, pp. 127-200.

JUDT, T. (2006). Postguerra: una historia de Europa desde 1945. Madrid: Ed. Taurus Santillana, pp. 725-727.

KEGLEY, C. W. \& WITTKOPF, I. E. (1999). World politics: trends and transformation. New York: Worth Publishers, pp. 382-425.

KISSINGER, H. (1979). Mis memorias. Buenos Aires: Ed. Atlántida, pp. 893-1010.

KORNBLOCH, P. (2004). Pinochet: los archivos secretos. Barcelona: Ed. Crítica, pp. 113-137.

LUNA, F. (1994). Breve historia de los Argentinos. Buenos Aires: Ed. Planeta, pp. 220-260.

MORGENTHAU, H. J. (1976). Politics among nations: the struggle for power and peace. New York: Ed. Alfred A. Knoff, pp. 225-262.

NEEDLER, M. (2009). National Security Doctrine: supply-side perspectives. In: INTERNATIONAL POLITICS SCIENCE ASSOCIATION, RESEARCH COMMITTEE. Armed Forces and Society: new challenges and environments. Santiago de Chile: Ed. Centro de Estudios e Investigaciones Militares (CESIM), pp. 505-514.

NIXON, R. (1990). En la arena. Barcelona: Ed. Plaza y Janés, pp. 358-367.

NYE, J. (2002). The paradox of American power: why the world only superpower can't go it alone. New York: Oxford University Press.

RAPOPORT, M. (2005). Historia económica, política y social de la Argentina 18802003. Buenos Aires: Editorial Ariel, pp. 309-415.

RAMOS ÁLVAREZ, M. (2009). The sociopolitical and institutional impact assessment of military technology in Venezuela. In: INTERNATIONAL POLITICS SCIENCE ASSOCIATION, RESEARCH COMMITTEE. Armed Forces and Society: new challenges and environments. Santiago de Chile: Ed. Centro de Estudios e Investigaciones Militares (CESIM), pp. 433-443.

ROBIN, M-M. (2005). Escuadrones de la muerte: la Escuela Francesa. Buenos Aires: Ed. Sudamericana, pp. 15-84. 
SPANIER, (1997). La política exterior norteamericana a partir de la Segunda Guerra Mundial. Buenos Aires: Grupo Editor Latinoamericano, pp. 299-310.

SOUAIDIA, H. (2002). La guerra sucia. Barcelona: Ediciones B.S.A.

UREDA, R. (2004). Muerte en el Pentagonito: Ios cementerios secretos del Ejército Peruano. Lima: Ed. Planeta, pp. 55-125.

VILLAMIZAR, E. (2007). Pensamiento estratégico contemporáneo y su aporte a la comprensión de los conflictos iberoamericanos no convencionales. En: CONTRERAS POLGATI, A.; DEMAREST, G. y GAETE PAREDES, J. (editores). Globalización, fenómenos transnacionales y seguridad hemisférica. Santiago de Chile: Instituto Histórico de Chile y Foreign Military Studies Office, pp. 359-377.

WEINER, T. (2008). Legado de cenizas: la historia de la CIA. Barcelona: Ed. Random House Mondadori, pp. 175-217.

WEST, N. (1997). La guerra secreta por las Malvinas: los Exocets y el espionaje internacional. Buenos Aires: Ed. Sudamericana, 1997.

ZAVERUCHA, J. (2009). Brazilian army performing the role of police: the emblematic case of Morro da Providencia. In: INTERNATIONAL POLITICS SCIENCE ASSOCIATION, RESEARCH COMMITTEE. Armed Forces and Society: new challenges and environments. Santiago de Chile: Ed. Centro de Estudios e Investigaciones Militares (CESIM), pp. 57-67.

ZERAOUI, Z. (2008). Seguridad internacional y terrorismo. En: CONSANI, N.; SEPÚLVEDA, A. y ZERAOUI, Z. (compiladores). Transitando los inicios del siglo XXI, las relaciones internacionales de Argentina, Chile y México. Santiago de Chile: Ril Editores, pp. 265-304. 